\title{
Carlo Gualtieri and Dragutin T. Mihailović (eds): Fluid Mechanics of Environmental Interfaces
}

\author{
Taylor and Francis, 2008, 332 pp. hardcover, price: \$169.95; ISBN \\ 978-0415-44669-3
}

\author{
Fabián A. Bombardelli · Kaveh Zamani
}

Received: 11 June 2009 / Accepted: 12 June 2009 / Published online: 4 July 2009

(C) The Author(s) 2009. This article is published with open access at Springerlink.com

The Environmental Fluid Mechanics (EFM) field addresses the motion of air and water at diverse scales, the fate and transport of species carried along by these fluids, and the interactions among those flows and geological, biological, and engineered systems. EFM emerged several decades ago as a response to the need of tools to study problems of flow and transport in rivers, estuaries, lakes, and the atmosphere; it is a topic of increasing concern for decision makers, engineers, and researchers alike. Several books have been published recently on EFM, but to the best of our knowledge none has dealt with the approach followed by this book.

This book, which has been contributed by distinguished researchers in the field, discusses the processes of mass, momentum, and heat exchange at various environmental interfaces (this is where the novelty lies). The audience of the book is vast, but it is constituted mainly by researchers. Some chapters of the book could be used as supplemental material for lecture notes in the first years of graduate school in civil and environmental engineering, environmental science, atmospheric science, meteorology, limnology, oceanography, and geophysics.

The book is organized in three parts with a total of ten chapters. Part one, which is composed of four chapters, covers the processes occurring at the interfaces of the atmosphere with deserts and seas. Part two deals in two chapters with the fluid mechanics of the interface between the atmosphere and water at smaller scales. Finally, part three discusses in four chapters the processes at the interfaces between fluids and biotic systems.

The first chapter was written by B. Cushman-Roisin, C. Gualtieri, and D. T. Mihailović. It acts as a presentation of the approach followed in the book and summarizes current knowledge in, and achievements of EFM. The chapter begins with an assessment of the importance of fluids for sustaining life on Earth. The chapter continues with discussions regarding two main ingredients of EFM, namely, turbulence, and stratification. The chapter also introduces the spatial and time scales at which EFM problems are investigated-from small eddies in vegetated channels to continental and global scales; from seconds up to decades, respectively. The

F. A. Bombardelli $(\bowtie) \cdot$ K. Zamani

Department of Civil and Environmental Engineering, University of California, Davis, 2001 Engineering

III, One Shields Ave., Davis, CA 95616, USA

e-mail: fabombardelli@ucdavis.edu 
authors finally delineate the set of "interfaces" in the environment (air-water, air-land, watersediment, and water-vegetation interface) and enumerate the challenges found in modelling those environmental interfaces.

Chapter two, contributed by B. Rajković, I. Arsenić, and Z. Grsić, covers theoretical aspects of the diffusion, advection, and sources/sinks of pollutants in the atmosphere. It starts by discussing the concepts associated with molecular and turbulent diffusion, and Fick's law; Taylor's theorem, and Richardson theory for turbulent diffusion follow. Point source, turbulent diffusion of passive scalars is then discussed, leading to Gaussian models; Puff models, which deal with non-stationary releases (customary under wind conditions), are presented afterwards. The chapter closes with a subsection on the parameterization of dry and wet deposition for non-conservative pollutants.

Chapter three, written by V. Djurdjević, and B. Rajkolic, deals with the air-sea interaction. At first, a characterization of the differences between boundary layers in water and air is presented. Then, concepts associated with the fluxes of mass, momentum, and heat between the ocean and the atmosphere are analysed. The chapter finishes with a description of a modelling experience regarding the sea surface temperature (SST) in the Mediterranean Sea, with satisfactory agreement with observations.

Chapter four was contributed by D. T. Mihailović and D. Kapor. It discusses exchanges between heterogeneous surfaces and the atmosphere, and assesses how to represent those fluxes at the cell scale in models. In dealing with variables in heterogeneous surfaces, issues associated with "aggregation" appear in a similar fashion to that occurring when one needs to spatially average in other fields of science. To address these issues, the following matters are discussed in this chapter: the concept of blending height (an approach which considers the distance of over which modifications of the air conditions are not altered by the surface), and alternative approaches for aggregation of aerodynamic surface parameters, including albedo, roughness length, surface temperature, and water-vapor pressure.

G. Kallos and P. Katsafados address in chapter five the important topic of desert dust uptake, transport, and deposition. The dust cycle is crucial in its ability to affect the radiation near the surface; it can also affect precipitation and biological processes. After a general description of the problem, the chapter analyses the main physical processes of the dust cycle, the settling velocity of particles, and the threshold friction velocity at which erosion starts; it additionally includes a section on dust source areas in Africa and their characteristics. The reader will find this chapter interesting as a means of opposing concepts of advection and diffusion in atmospheric settings versus waterborne flows, in terms of both complexity and scale. Wind fields and turbulence are analysed as key factors in the mobilisation of dust during unstable boundary-layer configurations in the daytime period, which then disappear at night. The chapter finishes with discussions on the surface concentration of dust and includes predictors for the sink of dust (dry and wet) from the atmosphere back to the surface.

Chapter six, prepared by C. Gualtieri and G. Pulci Doria, begins with an introductory section on general concepts regarding the transport processes which govern the gas transfer across air-water interfaces. The authors cover a wide variety of topics, ranging from the influence of physico-chemical characteristics of the gas being transferred on the transfer rate, to how turbulence interacts with the air-water interface and dictates the values of the gas-transfer coefficient. Different predictors for this coefficient are analysed in detail.

H. Chanson contributed chapter seven, which very conveniently supplements the previous chapter. After a nice description of the physical processes involved in air entrainment, the author uses analytical solutions of special advection-diffusion equations to approximate the distribution of void fraction in open-channel flow, plunging jets, hydraulic jumps, and jets issuing into air. The last section of the chapter is devoted to the description of experimental 
techniques applied to the observation of air concentration, bubble frequency, and turbulence in bubbly flows.

The third and last part of the book begins with chapter eight by D. T. Mihailović, which deals with the interaction between soil vegetation and the lower atmosphere. In particular, the chapter focuses on parameterization schemes of the interactions that take part in general circulation, mesoscale, and small-scale atmospheric numerical models.

Chapter nine, contributed by B. Lalić and D. T. Mihailović, starts by describing the different approaches to model the forest canopy structure based on the leaf area index (LAI) and the leaf area density (LAD) as key parameters. The chapter then discusses the velocity profiles above the forest and the exchange of momentum in the lower atmosphere. Finally, the authors devote space to discuss turbulence aspects within the forest. Many topics of this chapter serve as a nice introduction to the following chapter.

Chapter ten, by P. Gualtieri and G. Pulci Doria, starts with considerations of boundarylayer theory followed by a rather comprehensive review of recent studies on the mechanics of water flow in channels with submerged vegetation. In the second part of the chapter, the authors present their own experimental results on the subject using Laser Doppler Anemometry (LDA).

The last chapter, written by G. Nishihara and J. Ackerman, deals with processes of mass transfer to/from the surface of organisms in aquatic environments. The authors discuss momentum and concentration boundary layers over a model leaf, a topic which has numerous applications in science and engineering. The processes considered are for pelagic and benthic organisms. Benthic organisms play an important role in the spreading of pollutants through the water-sediment interface, thus becoming crucial actors in the cycling of metals.

Overall, "Fluid Mechanics of Environmental Interfaces" contributes to the field of EFM through a novel approach applied to a wide range of topics. The book is well organized, it is easy to read, and it has been published to high-quality standards. We believe that the book would have benefited from additional, specific chapters on "water-sediment interfaces," and "interfaces in water bodies" which appear as a result of stratification. In addition, a standalone appendix on turbulence would have provided the readers with more supporting material for better understanding the ideas conveyed in the chapters. Notwithstanding this suggestion, the book offers a wealth of information through up to date treatments of diverse topics of importance in EFM. The book allows for a direct comparison of concepts of diffusion, advection, and reaction in water and air, as well as the effect of vegetation on the flow in such environments. Finally, the book will undoubtedly be useful for researches in the field.

Open Access This article is distributed under the terms of the Creative Commons Attribution Noncommercial License which permits any noncommercial use, distribution, and reproduction in any medium, provided the original author(s) and source are credited. 